\title{
Biological control of Fusarium wilt of tomato by arbuscular mycorrhizal fungi with intercropping
}

\author{
Singh $\mathbf{M}^{1}$, Mishra $\mathbf{M}^{2}$, Srivastava $\mathrm{DK}^{3}$, Singh $\mathrm{PK}^{3^{*}}$ \\ ${ }^{1}$ Department of Environment Studies, Panjab University, Chandigarh - 160 014, India \\ ${ }^{2}$ International Centre for Genetic Engineering and Biotechnology, New Delhi - 110 067, India \\ ${ }^{3}$ Department of Botany, Eternal University, Baru-Sahib, Rajgarh, Sirmour - 173 101, HP, India
}

Singh M, Mishra M, Srivastava DK, Singh PK 2020 - Biological control of Fusarium wilt of tomato by arbuscular mycorrhizal fungi with intercropping. Plant Pathology \& Quarantine 10(1), 1-9, Doi 10.5943/ppq/10/1/1

\begin{abstract}
A pot experiment was carried to study the impact of the intercropping system i.e. tomato/tomato, tomato/maize, tomato/chilli and tomato/eggplant on interaction with Fusarium oxysporum f. sp. lycopersici (Fol) and arbuscular mycorrhizal (AM) fungi. AM fungi root colonization of tomato was significantly affected by the intercropping system. AM fungi inoculated plants expressed increase biomass (26.72 g shoot fresh weight \& 12.53 root fresh weight) of tomato compared to the untreated control (9.92 g root fresh weight \& $3.64 \mathrm{~g}$ root fresh weight) treatment. The intercropping crops maize, chilli, eggplant, and tomato had no effect on disease incidence or disease severity; however, tomato significantly showed a negative effect on one plant/pot with regard to biomass and disease severity of Fol co-cultivated with tomato. The results of the bioprotection effects of AM fungi observed in the decrease of disease severity and/or damage of plant biomass does not depend on the AM fungi colonization but more on the intercropping crops.
\end{abstract}

Key words - Bioprotection - Root Colonization - Mycorrhiza - Biotrophs - Soil-borne

\section{Introduction}

The properties of soil and plant-microbe interaction in the rhizosphere make it a unique and active area. About $80 \%$ of vascular plants root colonized with symbiotic soil fungi, arbuscular mycorrhizal (AM) fungi and enhances the growth and survival of numerous plant species (Smith \& Read 2008). AM fungi are obligate biotrophs, which infect plant roots for the exchange of carbon, pass on nutrients such as $\mathrm{P}, \mathrm{Fe}$, and $\mathrm{Zn}$ to the colonized root. Such relationships are mainly mutualistic, but can also be parasitic, depending on the fungal species and host plant involved (Singh \& Vyas 2009). AM fungi is reputed to control a number of plant diseases, especially soil-borne diseases (Singh et al. 2010a, b, 2014, Kabdwal et al. 2019) and it is known that they have an impact on plant community structure and diversity by altering inter or intra-specific competitive situations (van der Heijden et al. 2003, Singh et al. 2011). The symbiotic group of plants with AM fungi favour water and mineral nutrition and decrease abiotic and biotic stresses. (Jain \& Pundhir 2019). In return, the AM fungal community structure can be influenced by the host plants (Smith \& Read 2008). For the application of intercropping of tomato in combination with AMF and feedbacks between plants species mediated by AM fungi could alter the outcome of intercropping and therefore each intercropping arrangement should be tested separately. The application to crop species, especially 
with regard to intercropping arrangements with the aim of improved plant performance, includes lower infection rates causes by diseases (Hage-Ahmed et al. 2013). According to Ratnadass et al. (2012) not only AM fungi diversity but also plant species diversity could make a significant contribution to the reduction of plant diseases. Thus it could be intercropping utilizing by AM fungi reduce plant disease and the use of chemicals, and it reduces adverse effects on humans and the environment.

Fusarium oxysporum f. sp. lycopersici (Fol) is a soil-borne fungus, which attacks the plants through the roots and causes wilting in tomato which can result in severe yield losses (Singh et al. 2010b, Bidellaoui et al. 2019). The chemical control of soil-borne pathogens is difficult to control, so it gives strong reasons for alternative methods of disease control i.e. biological control or integrated disease management systems (Singh 2015, Jain \& Pundhir 2019). It has been shown by many workers previously that AM fungi reduced adverse effects of Fol in tomato when co-inoculated with this pathogen (Dehne \& Schonbeck 1979, Akköprü \& Demir 2005, Singh et al. 2010a, b, 2014, Singh 2015, Kabdwal et al. 2019). Additionally, suppression by root exudates of pathogens can occur in intercropping arrangements like for tomato and Chinese chive against Pseudomonas solanacearum (Yu 1999) and for watermelon and rice against Fusarium oxysporum f. sp. niveum (Hao et al. 2010). A combination of intercropping partners and the bioprotection effects of AM fungi can be considered as a new potential strategy against soil-borne pathogens and it would be the high significance for sustainable agriculture. The constitutive and inducible changes in morphological structures of root and secondary metabolism have direct and indirect interactions of hosts and their associated microbes (Philippot et al. 2013, Oldroyd 2013).

In our work, we focused on tomato (Solanum lycopersicon Mill. Cv. 'Sadabahar') intercropped with maize (Zea mays L., a model plant for work on AM fungi), chilli (Capsicum annum L.) and eggplant (Solanum melongena L.,) both members of the Solanaceae family as tomato in combination with AM fungal inocula. The aim was to study AM fungal root colonization of tomato in intercropping systems, $F$. oxysporum disease severity, and shoot and root weights of tomato.

\section{Materials \& Methods}

Tomato (Solanum lycopersicon Mill. cv. Sadabahar), maize (Zea mays L. cv. Vivek 27), Chilli (Capsicum annum L. cv. GT Sanam) and Eggplant (Solanum melongena L. cv. Shiva) were grown from seed surface sterilized by soaking in $5 \% \mathrm{NaOCl}$ for 5 minutes and rinsed with distilled water. Seeds were transferred to pots filled with autoclaved soil and incubated in a growth chamber with a $14 \mathrm{~h}$ light (light intensity $296 \mu \mathrm{mol} \mathrm{m} \mathrm{m}^{-1} \mathrm{~s}^{-1}$ ) and $10 \mathrm{~h}$ dark photoperiod at $25 \pm 1^{\circ} \mathrm{C}$. The soil was irrigated with distilled water. Seeds of tomato, maize, chilli, and eggplant were planted 3 weeks before transplanting.

Fusarium oxysporum f. sp. lycopersici was isolated from tomato variety, Sadabahar showing wilt characteristic symptom and the culture, Fol identification was made based on the characters depicted by Booth (1971). Fol was cultivated for 2 weeks at $27 \pm 1^{\circ} \mathrm{C}$ in darkness on Czapek Dox agar (HiMedia). The conidial suspensions were prepared as follows: mycelium was collected from 2 weeks old colonies grown on Czapek Dox agar with a scalpel and suspended in a sterile Czapek Dox broth (500 mg agar per liter). Suspensions were then filtered through sterilized cheesecloth (20-150 $\mu \mathrm{m}$ pore diameter) to obtain pure conidial suspensions and quantified by using a hemocytometer to a final concentration of $10^{6}$ microconidia $\mathrm{ml}^{-1}$. For AM fungal plant inoculation, a mixture of approximately 500 spores of Funneliformis mosseae and Rhizophagus irregularis obtained from The Energy Research Institute (TERI), New Delhi, INDIA.

Pre-cultivated plantlets of tomato, maize, chilli, and eggplant were transferred to pots $(24 \mathrm{~cm}$ diameter and $20 \mathrm{~cm}$ height) filled with an autoclaved ( $1 \mathrm{~h}$ at $121^{\circ} \mathrm{C}$ ) mixture of sand, soil and compost $(2: 1: 1, \mathrm{v} / \mathrm{v} / \mathrm{v})$. The plants were cultivated as a dual culture system with the following plant combinations: tomato/tomato, tomato/maize, tomato/chilli, and tomato/eggplant. The experimental set-up included four different treatments for each dual culture: (1) Fol, (2)AMF fungi, (3) Fol and AM fungi and (4) control without Fol and AM fungi. For each treatment 5 replicates comprising 4 pots each was used, giving 80 pots per plant combination and a total of 320 pots. For the AM fungi 
treatment, 200 spores of the AM fungi inoculum were added to the planting hole at the potting procedure. Fol was applied to the plant roots by dipping the roots for $5 \mathrm{~min}$ in a microconidial suspension $\left(10^{6}\right.$ microconidia $\left.\mathrm{ml}^{-1}\right)$ before plants were transferred to the pots. For the AM fungi + Fol treatment, both inocula were added as mentioned above. The plants were grown in a random design in a greenhouse for 13 weeks and were irrigated according to their moisture requirements with a half Hoagland nutrient solution (Hoagland \& Arnon 1938). After 13 weeks, the plants were gently removed from the substrate and washed thoroughly under tap water. Root and shoot fresh weights were determined. Plants of the tomato/tomato combination were separated into two groups per pot according to their root weights to assess intra-specific effects. Disease incidence was calculated according to the following formula:

$$
\text { Disease Incidence }=\frac{\text { Number of infected plants }}{\text { Total number of plants }} \times 100
$$

Fol disease severity was determined by measuring the amount of vessel discoloration of the stem in relation to the total stem length (length of infected stem $[\mathrm{cm}] /$ total length of stem $[\mathrm{cm}]$ ). Leaf symptoms were not evident at this plant stage and were therefore not considered for disease severity assessment. For confirmation of Fol infection, segments of $2 \mathrm{~cm}$ length starting upwards the shoot basis were dipped in $70 \%$ ethanol, flamed and put into Petri dishes containing potato dextrose agar amended with antibiotics to prevent bacterial growth according to Steinkellner et al. (2011). The Fol was estimated according to Nelson et al. (1983) by visual and microscopic analyses.

Defined root segments of $1 \mathrm{~cm}$ length, starting $2 \mathrm{~cm}$ down the shoot were used for determining the degree of mycorrhization. The root segments were cleared by boiling for $4 \min$ in $10 \% \mathrm{KOH}$ and afterward rinsed three times with tap water. Roots were stained by boiling for 3 min in a $5 \%$ inkvinegar solution (Vierheilig et al. 1998). The percentage of root colonization was determined according to the method of McGonigle et al. (1990).

\section{Results}

\section{Root Colonization}

The intercropping plants significantly influenced the AM fungal colonization in tomato plants (Fig. 1). Tomato plants intercropped with maize (TM) showed a $22.7 \%$ higher colonization than tomato co-cultivated with tomato (TT) whereas tomato intercropped with eggplant (TE) showed 13.3\% lower colonization. Tomato intercropped with chilli (TC) did not show any significant difference in the AM fungal colonization compared to the TT combination. Within TT combination, tomato plants grown in the same pot did not show significant difference colonization. Fol did not show an influence on AM fungal colonization compared to the AM fungal treatment alone. Tomato intercropped with maize (TM) showed a $22.7 \%$ higher colonization level than tomato intercropped with tomato and the other hand, while eggplant (TE) decreased the colonization level of tomato by $13.3 \%$. Chilli (TC) had a similar colonization level (74 \%) as its intercropping partner tomato.

\section{Disease Incidence and Severity}

Fusarium oxysporum f. sp. lycopersici (Fol) disease incidence and disease severity are showed in Fig. 2. For the TT combination, means of all plants from pots are given. Also, tomato plants of one pot were separated according to their root weights (Fig. 3) to see intraspecific effects and presented as $\mathrm{TT}^{\#}$ and $\mathrm{TT}^{\$}$. With regards to root weights of the tomato plants of one pot, disease incidence in the TT combination of $\mathrm{TT}^{\#}$ was reduced in the AM fungi + Fol treatment $(32.6 \pm 3.12 \%)$ and, consequently, showed almost 50\% less disease incidence than in the Fol treatment $(60.5 \%)$. Root and shoot weight increases were dependent on both AM fungi and intercropping system. Negative effects on tomato root or shoot weights due to AM fungi could not be observed in the present work. In the present work, two different AM fungal species $F$. mosseae and $R$. irregularis were inoculated together. 


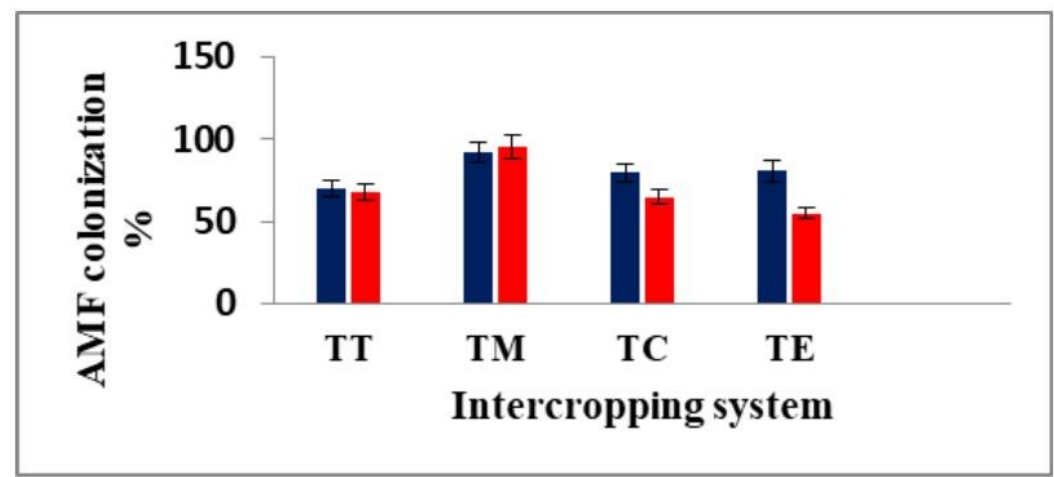

Fig. 1 - AM fungi mycorrhization (\%) in tomato roots with different intercropping partner plants. (TT = Tomato-Tomato; TM = Tomato-Maize; TC = Tomato-Chilli; TE = Tomato-Eggplant; Blue bar represents tomato while red represents partner crop).

Disease severity within the Fol treatment ranged between $12.4 \pm 0.94$ and $22.6 \pm 2.13 \%$ and between 5.8 \pm 0.45 and 20.6 $\pm 2.13 \%$ within the AM fungi + Fol treatment (Fig. 2). The plant combinations had neither in the Fol nor in the AM fungi + Fol treatment have a significant influence on Fol disease severity. The study of the influence of AM fungi and/or Fol on the growth of intercropped tomato showed that the intercropping partners of tomato impacted on root and shoot weight. Most striking was the reduction of 50 and $40 \%$, respectively, in the root and shoot weights of one tomato plant/pot in the TT control treatment compared to the other tomato plant. AM fungi did not change the effects of the intercropping partner on these growth parameters compared to the control treatment. Within the TC and TE combinations, AM fungi + Fol reduced disease severity significantly by $34.3 \%(\mathrm{P}<0.05)$ and $29.1 \%(\mathrm{P}<0.05)$, respectively. In the other plant combinations, the AM fungi + Fol treatment also tended to show lower disease severity. Within $\mathrm{TT}^{\$}$, Fol showed higher disease severity than AM fungi + Fol $(\mathrm{P}<0.05)$. Within $\mathrm{TT}^{\#}$, disease severity was similar between Fol and AM fungi + Fol. Within the Fol $(\mathrm{P}<0.05)$ as well as in the AM fungi + Fol $(\mathrm{P}<0.05)$ column, $\mathrm{TT}^{\#}$ had significantly lower disease severity than $\mathrm{TT}^{\$}$.



Fig. 2 - Disease incidence $(\%)$ and severity $(\%)$ in tomato under the Fol and AM fungi + Fol treatments. $\left(\mathrm{TT}^{*}=\right.$ Stronger and healthy tomato-Weaker and unhealthy tomato; $\mathrm{TT}^{\#}=$ TomatoStronger and healthy tomato; $\mathrm{TT}^{\$}=$ Tomato-Weaker and unhealthy tomato; $\mathrm{TM}=$ Tomato-Maize; $\mathrm{TC}=$ Tomato-Chilli; $\mathrm{TE}=$ Tomato-Eggplant).

\section{Plant Growth}

Plant growth of tomato in the different plant combinations and treatments was assessed by root and shoot weights. Root weights for the treatments with Fol and/or AM fungal application within the different plant combinations are shown in Fig. 3. The intercropping system had in each treatment a 
significant effect on tomato root weights (Fig. 3). Root weights of $\mathrm{TT}^{\#}$ and $\mathrm{TT}^{\$}$ of the $\mathrm{TT}$ combination in the control treatment differed significantly, indicating an intraspecific effect of tomato plants. Plants of $\mathrm{TT}^{\$}$ had almost $50 \%$ less weight than the ones from $\mathrm{TT}^{\#}$. $\mathrm{TM}$ and $\mathrm{TC}$ showed the highest tomato root weights, whilst TC ranged between the lowest and the highest root weights. These trends were also seen in the Fol, AM fungi and AM fungi + Fol treatments.

With regard to root weight of tomato plants in one pot, disease incidence in the TT combination was reduced in the AM fungi + Fol (Fig. 4). Within the control treatment, TT\$ of the TT combination showed around $33 \%$ less shoot weight than $\mathrm{TT}^{\#}$. The highest shoot weights were reached by tomato plants of the TM combination (increases up to 20 and $47 \%$, compared to $\mathrm{TT}^{\#}$ and $\mathrm{TT}^{\$}$, respectively). The shoot weights of the tomato plants of the TC and TE combinations ranged between TT and TM.

When assessing the influence of AMF inoculation in intercropping systems on Fol disease severity in tomato, a bioprotective effect was observed. This bioprotective effect resulted in reduced disease severity in AM fungal plants of the TT" ${ }^{\#}, \mathrm{TC}, \mathrm{TE}$ and TM treatments. Looking on $\mathrm{TT}^{\#}$, TM, and TC, AM fungi + Fol treated plants produced more shoot biomass compared to the Fol treated plants. Thus, mycorrhization enhances the tolerance of the tomato plants to the pathogen. Positive effects of AM fungi expressed as an increase in biomass compared to the control treatment, could be observed for root as well as shoot weights.



Fig. 3 - Tomato root weight (g) under different (Control, Fol, AM fungi, AM fungi + Fol) treatments. $\left(\mathrm{TT}^{\#}=\right.$ Tomato-Stronger and healthy tomato; $\mathrm{TT}^{\$}=$ Tomato-Weaker and unhealthy tomato; $\mathrm{TM}=$ Tomato-Maize; $\mathrm{TC}=$ Tomato-Chilli; $\mathrm{TE}=$ Tomato-Eggplant $)$.

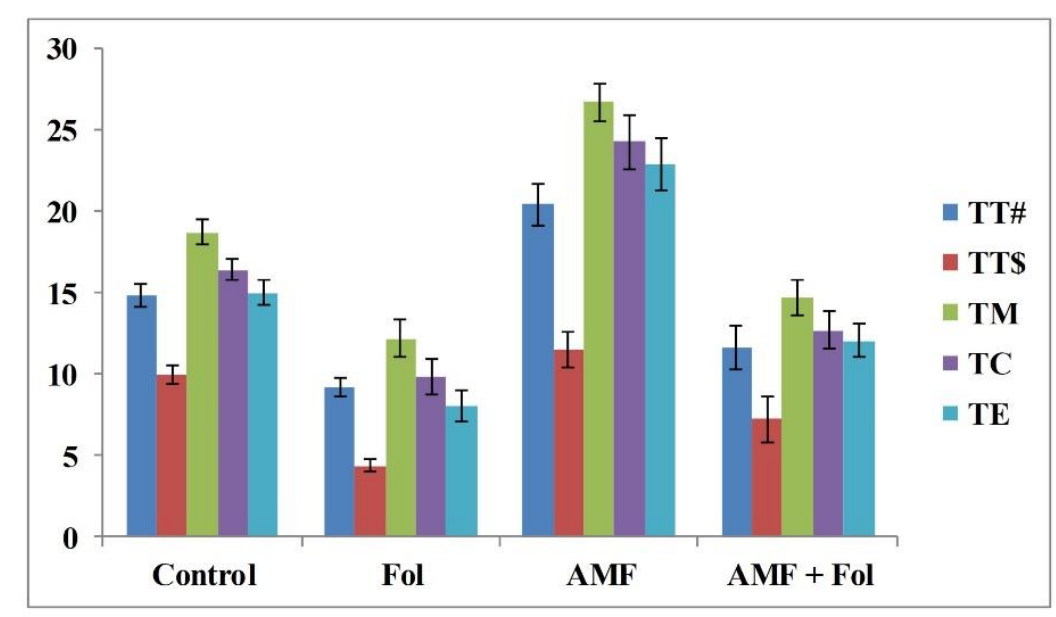

Fig. 4 - Tomato shoot weight (g) under different (Control, Fol, AM fungi, AM fungi + Fol) treatments. $\left(\mathrm{TT}^{\#}=\right.$ Tomato-Stronger and healthy tomato; $\mathrm{TT}^{\$}=$ Tomato-Weaker and unhealthy tomato; $\mathrm{TM}=$ Tomato-Maize; $\mathrm{TC}=$ Tomato-Chilli; $\mathrm{TE}=$ Tomato-Eggplant). 


\section{Discussion}

Their bioprotecion role and characteristics of AM fungi are of great interest in the perspective of sustainable agriculture. The benefit of AM fungi with the utilization of plant species diversity shows great promise for the management of plant diseases in environmentally compatible agriculture. In this study, tomato intercropped with maize, chilli, eggplant or tomato itself with AM fungi were tested against Fol. Plants of the control and Fol treated were also checked for AM fungi colonization but they did not show any presence of AM fungi. It has been well documented that increasing crop species diversity can yield benefits in plant disease control (Zhu et al. 2000). However, most studies have focused on above-ground plant functions and performances. Few plant disease studies pay attention to the root, the main organ of plants that sense environmental attacks and related signals in the soil (Hage-Ahmed et al. 2013, Gao et al. 2014).

AM fungal symbiosis with its aspects of bio-fertilizer and bioprotection are the special interest in the context of sustainable agriculture (Singh \& Vyas 2009). According to Gao et al. (2014), wellestablished AM fungal symbiosis in maize stimulated the colonization of tomato and additionally, is in line with the frequent use of maize as nurse plants in experimental set-ups. In the other dual cultures, AM fungi + Fol also showed lower disease incidences than the Fol treatment alone. The plant combinations had no impact on Fol disease incidence. Root and shoot weight increases were dependent on both AM fungi and intercropping system. The results were contradictory that AM fungi had negative effects on the root as well as on the shoot weights of chilli, maize and zucchini grown in intra-specific density settings (Schroeder-Moreno \& Janos 2008). The negative effects on tomato root or shoot weights due to AM fungi could not be observed, with regards to inter-specific competition the AM fungal species can affect the outcome of a competitive situation between plants of Brachypodium pinnatum and Prunella vulgaris observed that the way in which the two plants coexisted depended on the Glomus spp. inoculated (van der Heijden et al. 2013).

In the present work, two different AM fungal species $F$. mosseae and $R$. irregularis were inoculated together and compensation by AM fungi for the negative effects of Fol on plant biomass could particularly be reached with eggplant as an intercropping partner. Thus, a well-chosen intercropping partner like eggplant and maize can allow expression of a bioprotection effect of AM fungi, even when the symbiosis is not established before pathogen inoculation. A different experiment, where AM fungi were applied before $F$. oxysporum $\mathrm{f}$. sp. ciceris inoculation might have led to more positive effects (Singh et al. 2010c, 2013). Positive effects of AM fungi expressed as an increase in biomass compared to the control treatment, could be observed for root and shoot weights. However, a simultaneous inoculation in an intercropping setting appears to be more comparable to natural conditions where the concurrent activity of AM fungi and Fol will not be uncommon (HageAhmed et al. 2013). Plant combinations had no significant influence on Fol disease severity and the intercropping partner of tomato impacted root and shoot weight. Besides the plant nutrient uptake, competition for space, nutrients, changes in the root system, mycorrhizosphere effect and the activation of plant defense mechanisms are responsible for disease inhibition by AM fungi.

The 'weaker' (tomato + tomato) partner also showed significantly higher Fol disease severity and no positive effects of AM fungi inoculation so it concluded that the positive effects of AM fungi on disease severity are limited in competitive situations and that the intercropping partner affects the positive effects of AM fungi on tomato plants with regard Fol disease severity (Hage-Ahmed et al. 2014). In addition, the crucial effects of the intercropping partner on AM fungi colonization of tomato were found, which is of great interest in crop plant communities and the influences on each other. However, the outcome of the AM fungi affects Fol disease severity and/or plant biomass did not depend on the degree of AM fungal colonization but more on the intercropping partner.

\section{Conclusions}

The present study demonstrates the intercropping system with tomato and its effects on the control of Fusarium wilt by AM fungi. Moreover, suitable intercrop combinations increase both plant growth and resistance to pathogens. Tomato plants intercropped with different species had no effect on Fol disease incidence or disease severity signifying no allelopathic suppression and tomato 
intercropped with tomato clearly showed negative effects on one plant/pot with observed to biomass and disease severity of Fol. Whereas also the crucial effects of the intercropping partner on AM fungi colonization of tomato were found, which is of great interest in crop plant communities and the influences on each other. However, the result of the AM fungal effects on Fol disease severity and/or plant biomass did not depend on the degree of AM fungi colonization but more on the intercropping partner. Taproot system of plants will be dependent on AM fungi than plants with fibrous root system and the environmental conditions can be modify the taproot plants and plants with fibrous root system through AM fungi functioning, which will regulate plant defense systems (Yang et al. 2014). In future studies, therefore more detailed investigations of the relationships in various intercrop systems and of the interactions between the micro-organisms and the host plant are needed for further understanding of the biocontrol of the related diseases.

\section{Acknowledgements}

PKS and MS thanks to the SERB, Department of Science and Technology, Government of India for awarding Fast Track Young Scientist and financial assistance. There is no conflict of interest between authors.

\section{References}

Akköprü A, Demir S. 2005 - Biological control of Fusarium wilt in tomato caused by Fusarium oxysporum f. sp. lycopersici by AMF Glomus intraradices and some rhizobacteria. Journal of Phytopathology 153, 544-550.

Bidellaoui B, Segarra G, Hakkou A, Trilas MI. 2019 - Beneficial effects of Rhizophagus irregularis and Trichoderma asperellum strain T34 on growth and Fusarium wilt in tomato plants. Journal of Plant Pathology, 101, 121-127.

Booth C, Mordue JE, Gibson IAS. 1971 - Description of pathogenic fungi and bacteria. Common Wealth Mycological Institute, 58, 571-580.

Dehne HW, Schonbeck F. 1979 - Untersuchungen zum Einfluß der endotrophen Mycorrhiza auf Pflanzenkrankheiten. Phytopathology Z 95, 105-110.

Gao X, Wu M, Xu R, Wang X et al. 2014 - Root interactions in a maize/soybean intercropping system control soybean soil-borne disease, red crown rot. PLoS ONE (5): e95031. doi:10.1371/journal.pone.0095031.

Hage-Ahmed K, Krammer J, Steinkellner S. 2013a - The intercropping partner affects arbuscular mycorrhizal fungi and Fusarium oxysporum f. sp. lycopersici interactions in tomato. Mycorrhiza 23, 543-550.

Hage-Ahmed K, Moyses A, Voglgruber A, Hadacek F, Steinkellner S. 2013b - Alternations in root exudation of intercropped tomato mediated by the arbuscular mycorrhizal fungus Glomus mosseae and the soil borne pathogen Fusarium oxysporum f. sp. lycopersici. Journal of Phytopathology 161, 763-773.

Hao W, Ren LX, Ran W, Shen QR. 2010 - Allelopathic effects of root exudates from watermelon and rice plants on Fusarium oxysporum f. sp. niveum. Plant Soil 336, 485-497.

Hoagland DR, Arnon DI. 1938 - The water-culture method for growing plants without soil, vol. 347. California Agricultural Experimental Station, Berkeley, Circular, pp. 1-39.

Jain P, Pundir RK. 2019 - Biocontrol of soil phytopathogens by arbuscular mycorrhiza - A review. In: Varma A., Choudhary D. (eds) Mycorrhizosphere and Pedogenesis. Springer, Singapore, pp. 221-237.

Kabdwal BC, Sharma R, Tewari R, Tewari AK et al. 2019 - Field efficacy of different combinations of Trichoderma harzianum, Pseudomonas fluorescens, and arbuscular mycorrhiza fungus against the major diseases of tomato in Uttarakhand (India). Egyptian Journal of Biological Pest Control 29, 1-10. 
McGonigle T, Miller M, Evans D, Fairchild G, Swan J. 1990 - A new method which gives an objective measure of colonization of roots by vesicular-arbuscular mycorrhizal fungi. New Phytologist 115, 495-501.

Nelson P, Toussoun T, Marasas W. 1983 - Fusarium species. An illustrated manual for identification. State University Press, University Park, Penn.

Oldroyd GED. 2013 - Speak, friend, and enter: signalling systems that promote beneficial symbiotic associations in plants. Nature Review Microbiology 11, 252-263.

Philippot L, Raaijmakers J, Lemanceau P, van der Putten WH. 2013 - Going back to the roots: the microbial ecology of the rhizosphere. Nature Review Microbiology 11, 789-799.

Ratnadass A, Fernandes P, Avelino J, Habib R. 2012 - Plant species diversity for sustainable management of crop pests and diseases in agroecosystems: a review. Agronomy and Sustainable Development 32, 273-303.

Schroeder-Moreno MS, Janos DP. 2008 - Intra- and inter-specific density affects plant growth responses to arbuscular mycorrhizas. Botany 86, 1180-1193.

Singh M, Singh PK, Vyas D. 2011 - Mycorrhization in medicinal plants. Mycorrhiza News 23, 1923.

Singh M, Vyas D, Singh PK. 2014 - Interaction of soil microbes with mycorrhizal fungi in tomato. Archives of Phytopathology and Plant Protection 47, 737-743. DOI: 10.1080/03235408. 2013.820389.

Singh M. 2015 - Interactions among arbuscular mycorrhizal fungi, Trichoderma harzianum, Aspergillus niger and biocontrol of wilt of tomato. Archives of Phytopathology and Plant Protection 48, 205-211. DOI: 10.1080/03235408.2014.884825.

Singh PK, Singh M, Agnihotri VK, Vyas D. 2013 - Arbuscular Mycorrhizal Fungi: Biocontrol against Fusarium Wilt of Chickpea. International Journal of Scientific Research and Publication 3, 1-5.

Singh PK, Singh M, Vyas D. 2010a - Biocontrol of Fusarium Wilt of Chickpea using Arbuscular Mycorrhizal Fungi and Rhizobium leguminosorum Biovar. Caryologia 63, 349-353.

Singh PK, Singh M, Vyas D. 2010b - Effect of root exudates of mycorrhizal tomato plants on microconidia germination of Fusarium oxysporum f. sp. lycopersici than root exudates from non-mycorrhizal tomato plants. Archives of Phytopathology and Plant Protection 43, 14951503.

Singh PK, Singh M, Vyas D. 2010c - Interactions of vesicular-arbuscular mycorrhizal fungi with Fusarium wilt and growth of tomato (Lycopersicon esculentum Mill.). Indian Phytopathology 63, 30-34.

Singh PK, Vyas D. 2009 - Biocontrol of plant diseases and sustainable agriculture. Proceedings of National Academy of Sciences India 79, 110-128.

Smith SE, Read DJ. 2008 - Mycorrhizal symbiosis, 3rd edn. Academic, London.

Steinkellner S, Hage-Ahmed K, Garcia-Garrido JM, Illana A et al. 2011 - A comparison of wildtype, old and modern tomato cultivars in the interaction with the arbuscular mycorrhizal fungus Glomus mosseae and the tomato pathogen Fusarium oxysporum f. sp. lycopersici. Mycorrhiza 22, 189-194.

van der Heijden M, Wiemken A, Sanders I. 2003 - Different arbuscular mycorrhizal fungi alter coexistence and resource distribution between co-occurring plant. New Phytologist 157, 569578.

Vierheilig H, Coughlan AP, Wyss U, Piche Y. 1998 - Ink and vinegar, a simple staining technique for arbuscular-mycorrhizal fungi. Applied and Environmental Microbiology 64, 5004-5007.

Yang H, Zhang Q, Dai Y, Liu Q et al. 2015 - Effects of arbuscular mycorrhizal fungi on plant growth depend on root system: a meta-analysis. Plant Soil 389, 361-374.

Yu JQ. 1999 - Allelopathic suppression of Pseudomonas solanacearum infection of tomato (Lycopersicon esculentum) in a tomato Chinese chive (Allium tuberosum) intercropping system. Journal of Chemical Ecology 25, 2409-2417. 
Zhu Y, Chen H, Fan J, Wang Y et al. 2000 - Genetic diversity and disease control in rice. Nature 406, 718-722. 